$\mathrm{DOE} / \mathrm{ER} / 25036--5$

DE92 006417

\title{
THE STUDY OF EFFECTS OF SMALL PERTURBATIONS ON CHAOTIC SYSTEMS
}

Progress Report

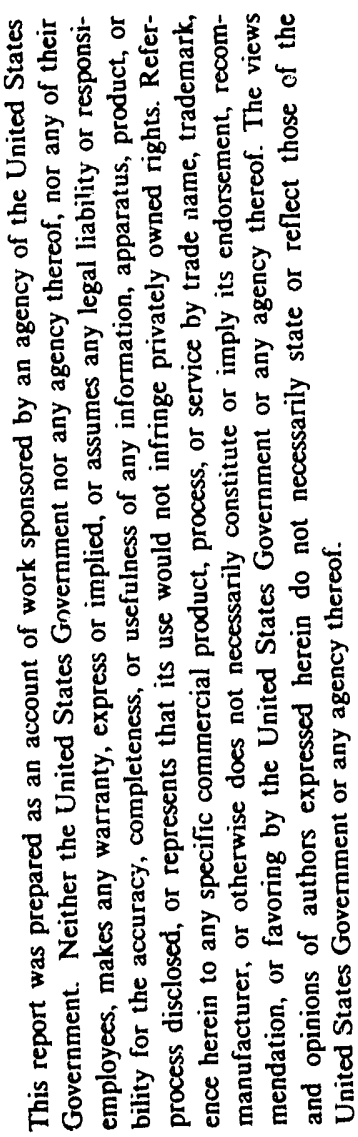

January 1991-December 1991

Celso Grebogi

Laboratory for Plasma Research

University of Maryland

College Park, MD 20742-3511

James A. Yorke

Institute for Physical Science and Technology

University of Maryland

College Park, MD 20742-2431

December 1991

Prepared for the U.S. Department of Energy

Under Grant Nurnber DE-FG05-87ER25036 


\section{INTRODUCTION}

The following report summarizes our iesearch activities under the U.S. Department of Energy, Scientific Computing Staff Office of Energy Research, Contract No. DE-FG05-87ER25036. We have organized the report under the following categories:

I. Work Done in the Period January 1991 Through December 1991.

A. Summary of the research done.

B. Papers published in refereed journals or refereed proceedings.

II. Planned work for the period January 1992 through December 1992.

III. Appendix reprints.

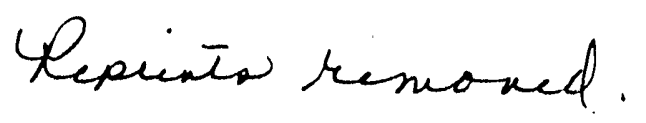




\section{WORK DONE IN THE PERIOD JANUARY 1991 THROUGH DECEMBER 1991}

\section{A. Summary of the Research Done}

\section{1. "Controlling Chaotic Dynamical Systems"}

The control of chaos is a major component in our DOE funded research program. In this work we found a method that converts the motion on a chaotic attractor to a desired attracting time periodic motion by making only small time dependent perturbations of a control parameter. The time periodic motion results from the stabilization of one of the infinite number of previously unstable periodic orbits embedded in the attractor. The present work extended that of Ott, Grebogi, and. Yorke [Phys. Rev. Lett. 64, 1196 (1990)], allowing for a more general choice of the feedback matrix and implementation to higher-dimensional systems. The method is illustrated by an application to the control of a periodically impulsively kicked dissipative mechanical system with two degrees of freedom resulting in a fourdimensional map (the "double rotor map"). A key issue addressed is that of the dependence of the average time to achieve control on the size of the perturbations and on the choice of the feedback matrix. Some comments concerning this method are the following:

1. Before settling into the desired controlled orbit the trajectory experiences a chaotic transient whose expected duration diverges as the maximum allowed size of the control approaches zero.

2. Small noise can result in occasional bursts in which the orbit wanders far from the controlled orbit.

3. Controlled chaotic systems offer an advantage in flexibulity in that any one of a number of orbits can be stabilized by the small control, and the choice can be switched from one to another depending on the current desired system performance. 


\section{2. "Eınbedding of Experimental Data"}

In this work we addressed fundamental mathematical formulations in embedding methods used for the reconstruction of attractors from experimental data. Embedding theorems, based on previous work by H. Whitney and F. Takens, are established for compact subsets $\mathrm{A}$ of Euclidean space $R^{k}$. If $\mathrm{n}$ is an integer larger than twice the box-counting dimension of $\mathrm{A}$, then almost every map from $R^{k}$ to $R^{n}$, in the sense of prevalence, is one-to-one on $\mathrm{A}$, and moreover is an embedding on smooth manifolds contained within $\mathrm{A}$. If $\mathrm{A}$ is a chaotic attractor of a typical dynamical system, then the same is true for almost every delay-coordinate map from $R^{k}$ to $R^{n}$. These results are extended in two other directions. Similar results are proved in the more general case of reconstructions which use moving averages of delay coordinates. Second, information is given on the self-intersection set that exists wien $\mathrm{n}$ is less than or equal to twice the boy counting dimension of $\mathrm{A}$.

\section{3. "Effect of Noise on Critical Exponents of Crises"}

The work on crises and chactic transients were developed under DOE contract. In this work, we considered the influence of random noise on low-dimensional, nonlinear dynamical systems with parameters near values leading to a crisis in the absence of noise. In a crisis, one of several characteristic changes in a chaotic attractor takes place as a system parameter $p$ passes through its crisis value $p_{c}$. For each type of change, there is a characteristic temporal behavior of orbits after the crisis ( $p>p_{c}$ by convention), with a characteristic time scale $\tau$. For an important class of deterministic systems, the dependence of $\tau$ on $p$ is $\tau \sim\left(p-p_{c}\right)^{-\gamma}$ for $p$ slightly greater than $p_{c}$. When noise is added to a system with $p<p_{c}$, orbits can exhibit the same sorts of characteristic temporal behavior as in the deterministic case for $p>p_{c}$ (a noise-induced crisis). Our main result is that for systems whose characteristic times scale as above in the zero-noise limit, the characteristic time in the noisy case scales as $\tau \sim \sigma^{-\gamma} g\left(\left(p_{c}-p\right) / \sigma\right)$, where $\sigma$ is the characteristic strength of the noise, $g(\cdot)$ is a nonuniversal function depending on the system and noise, 
and $\gamma$ is the critical exponent of the corresponding deterministic crisis. Illustrative numerical examples are given for two-dimensional maps and a three-dimensional flow. In addition, the relevance of the noise scaling law to experimental situations is discussed.

We also investigated experimentally the scaling of the average time $\tau$ between intermittent, noise-induced bursts for a chaotic mechanical system near a crisis. The system studied is a periodically driven (frequency $f$ ) magnetoelastic ribbon. Theory predicts that for deterministic crises where $\tau$ scales as $\tau \sim\left|f^{s}-f_{c}\right|^{-\tau}\left(f<f_{c}, f=f_{c}\right.$ at crisis), the characteristic time between noise-induced bursts $\left(f \geq f_{c}\right)$ should scale as $\tau \sim \sigma^{-r} g\left(\left|f-f_{c}\right| / \sigma\right)$ where $\sigma$ is the noise strength and $\gamma$ is the same in both cases. We determine $\gamma$ for the low-noise ("deterministic") system, then add noise and observe that the scaling for $\tau$ is as predicted.

\section{4. "Transition to Chaotic Scattering"}

Another major topic developed under DOE grant has been related with chaotic scattering. In this work we addressed the question of how chaotic scattering arises and evolves as a system parameter is continuously varied starting from a value for which the scattering is regular (i.e., not chaotic). Our results show that the transition from regular to chaotic scattering can occur via a saddle-center bifurcation, with further qualitative changes in the chaotic set resulting from a sequence of homoclinic and heteroclinic intersections. We also show that a state of "fully developed" chaotic scattering can be reached in our system through a process analogous to the formation of a Smale horseshoe. By fully developed chaotic scattering, we mean that the chaotic-invariant set is hyperbolic, and we find for our problem that all bounded orbits can be coded by a full shift on three symbols. Observable consequences related to qualitative changes in the chaotic set are also discussed.

\section{5. "Distribution of Floaters on a Fluid Surface"}

The long-time spatial distribution of particles floating on the surface of a confined fluid whose flow velocity has complicated time dependence was considered. It 
was shown that this distribution can be either a fractal or else can clump at several (or one) discrete points. The transition from the latter type of distribution to the former occurs when the Lyapunov exponent characterizing the particle motion passes through zero from negative values to positive values. The characteristic features of this type of transition are investigated using random maps. It was shown that near the transition there are extremely intermittent temporal fluctuations in the particle cloud, and their scaling with a parameter is elucidated. 


\section{B. Papers Published in Refereed Journals or Refereed Proceedings}

1. "Controlling Chaotic Dynamical Systems," F. J. Romeiras, E. Ott, C. Grebogi, and W. P. Dayawansa, Proc. 1991 American Control Conference (American Automatic Control Council, IEEE Service Center, Piscataway, NJ, 1991), pp. 1112-1119.

2. "Embedology," T. Sauer, J. A. Yorke, and M. Casdagli, J. Stat. Phys. 65, 579-616 (1991).

3. "Scaling Law for Characteristic Times of Noise-Induced Crises," J. C. Sommerer, E. Ott, and C. Grebogi, Phys. Rev. A 43, 1754-1769 (1991).

4. "Experimental Confirmation of the Scaling Theory for Noise-Induced Crises," J. C. Sommerer, W. L. Ditto, C. Grebogi, E. Ott, and M. L. Spano, Phys. Rev. Lett. 66, 1947-1950 (1991).

5. "Experimental Confirmation of the Theory for Critical Exponent of Crises," J. C. Sommerer, W. L. Ditto, C. Grebogi, E. Ott, and M. L. Spano, Phys. Lett. 153A, 105-109 (1991).

6. "Transition to Chaotic Scattering," M. Ding, C. Grebogi, E. Ott, and J. A. Yorke, Phys. Rev. A 42, 7025-7040 (1991).

7. "Massive Bifurcation of Chaotic Scattering," Phys. Lett. 153A, 21-26 (1991).

8. "Fractal Distribution of Floaters on a Fluid Surface and the Transition to Chaos for Random Maps," L. Yu, E. Ott, and Q. Chen, Physica 53D, 102$124(1991)$.

9. "The Spectrum of Fractal Dimensions of Passively Convected Scalar Gradients in Chaotic Fluid Flows," F. Varosi, T. M. Antonsen, and E. Ott, Phys. Fluids A 3, 1017-1028 (1991). 
10. "Calculating Topological Entropies of Chaotic Dynamical Systems," Q. Chen, E. Ott, and L. P. Hurd, Phys. Lett. 156A, 48-52 (1991).

11. "Cubic Maps as Models of Two-Dimensional Antimonotonicity," S. P. Dawson and C. Grebogi, Chaos, Solitons and Fractals, 1, 137-144 (1991). 


\section{PLANNED WORK FOR THE PERIOD JANUARY 1991 THROUGH DECEMBER 1991}

\section{1. "Prevalence"}

We introduced a measure-theoretic condition for a property to hold "almost everywhere" on an infinite dimensional vector space, with particular emphasis on function spaces such as $C^{k}$ and $L^{p}$. Like the concept of "Lebesgue almost every" on finite dimensional spaces, our notion of "prevalence" is translation invariant. Instead of using a particular measure on the entire space, we define prevalence in terms of the class of all probability measures with compact support. Prevalence is a more appropriate condition than the topological concepts of "open and dense" or "generic" when one desires a probabilities result on the likelihood of a given property on a function space. We give several examples of properties which hold "almost everywhere" in the sense of prevalence. For instance, we prove that almost every $C^{1}$ map on $R^{n}$ has the property that all of its periodic orbits are hyperbolic. We believe, this is a path-breaking concept that needs much further work.

\section{2. "Border Crossing Bifurcations"}

We intend to investigate the phenomenon of border bifurcation. These occur in piecewise smooth systems, which in turn occur in a variety of situations involving optimization and/or constraints. In the paper above, we have presented qualitatively different types of border bifurcations for piecewise smooth maps. L.gree theory, which is a useful tool analyzing bifurcation problems involving periodic orbits only, cannot always be applied as the examples show. Therefore, new methods have to be developed to attack the problem of border bifurcation phenomenon.

\section{3. "Numerical Procedure for Computing the Dimension"}

Based on our experience with the development of saddle straddle trajectories and the equality for Hausdorff, box-counting and uncertainty dimension, we intend to develop a numerical procedure for computing the dimension of one dimensionally 
unstable attracior. We will show that the method is guaranteed to work for a class of one-dimensionally unstable hyperbolic attractors.

\section{4. "Generalized Saddle Straddle Trajectories Methods"}

In many systems one observes transient chaos. This is due to the presence of chaotic saddles in the system. So far we have a numerical method (SST method) that generates a trajectory on a chaotic saddle (that is, a chaotic set that is not attracting) that has been implemented in the Yorke's DYNAMICS problem. We have shown that the SST method is guaranteed to work for a class of one-dimensionally unstable saddle-hyperbolic systems.

We intend to develop numerical methods for finding trajectories on chaotic saddles which are either at least two-dimensionally unstable sacifle-hyperbolic sets or include points that have a different unstable dimension. A problem related to shadowng and our SST method is the following. Assume that for $\downarrow$ given system. the SST method generates a numerical trajectory that can be computed as long as one wishes. Is it true that the numerical trajectory can be shadowed by a true trajectory?

\section{5. "Finding Typical Tra"ectories Numerically"}

Finding typical numerical trajectories is a central problem of simulating of chaotic systems. A fundamental question is whether or not the behavior of a typical trajectory is compatible with the behavior of some numerically generated trajectory. For a variety of problems, we are not interested in tracking a particular trajectory but rather we need information about the behavior of typical trajectories. In such rases, the problem becomes first of showing that numerical trajectories are shadowed by true trajectories, and second, that these true trajectories are typical. We believe these true trajectories are usually in fact "typical". Examples are common where the specific initial condition is not important, but the long term behavior is important. There are examples, where one wants to know the statistical behavior.

We plan to continue investigating a theoretical foundation for methods pro- 
ducing typical numerical trajectories. The different methods for different systems might vary slightly, depending on some global properties of the system that is considered. Our objective is to find a numerical procedure which produces a numerical trajectory which has the properties (1) the trajectory is shadowable, and (2) the trajectory is distributed throughout the attractor in accord with the natural measure. Of course, the latter statement will iave to be made precise, since only a finite number of iterates will be available. We believe we have a procedure that will work for low dimensional cl.aotic systems, but proving that it will work will be difficult. Our objective will be first to prove that it works for uniformly hyperbolic attractors. Some progress has been made on investigating some particular systems, such as maps near the map $x_{n+1}=3 x_{n} \bmod 1$. We propose to develop methods that are guaranteed to generate (nearly) typical numerical trajectories in a variety of d"namical systems. Furthermore we want to be able to obtain useful estimates for how close our trajectories are to being typical. 

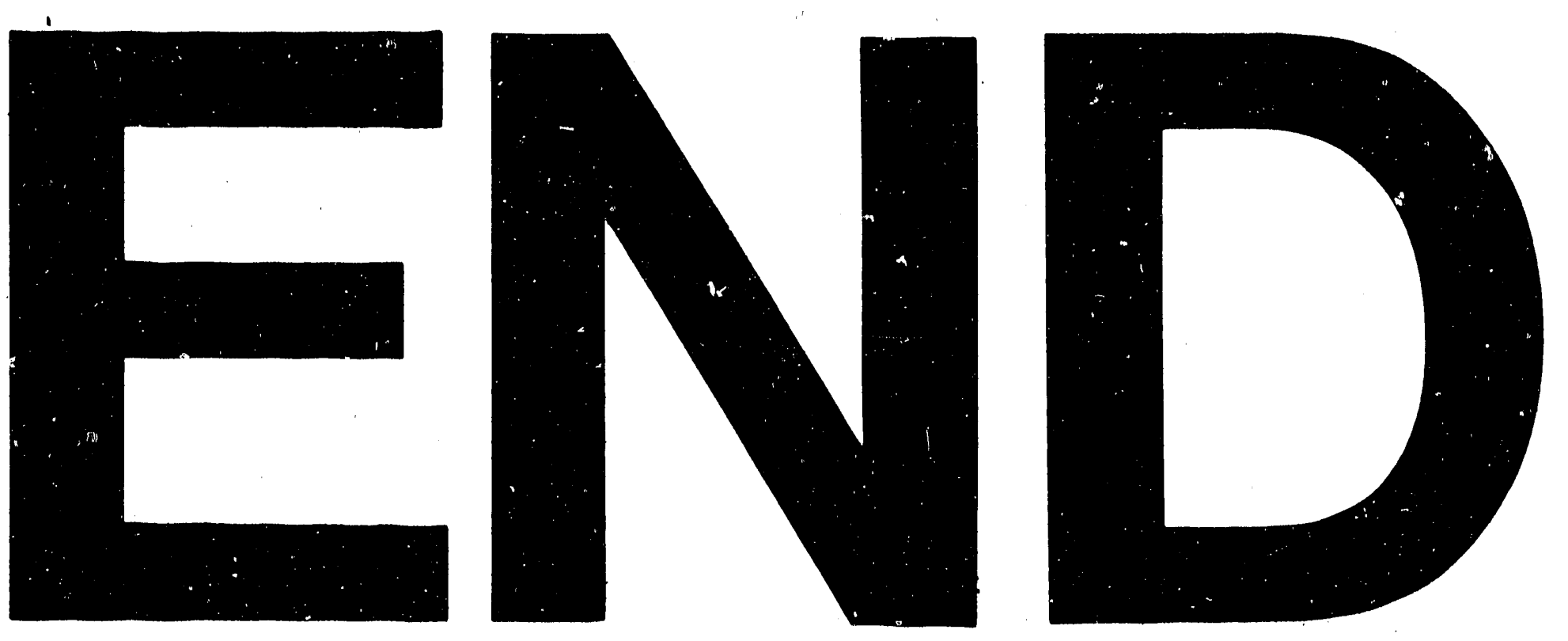

$-$
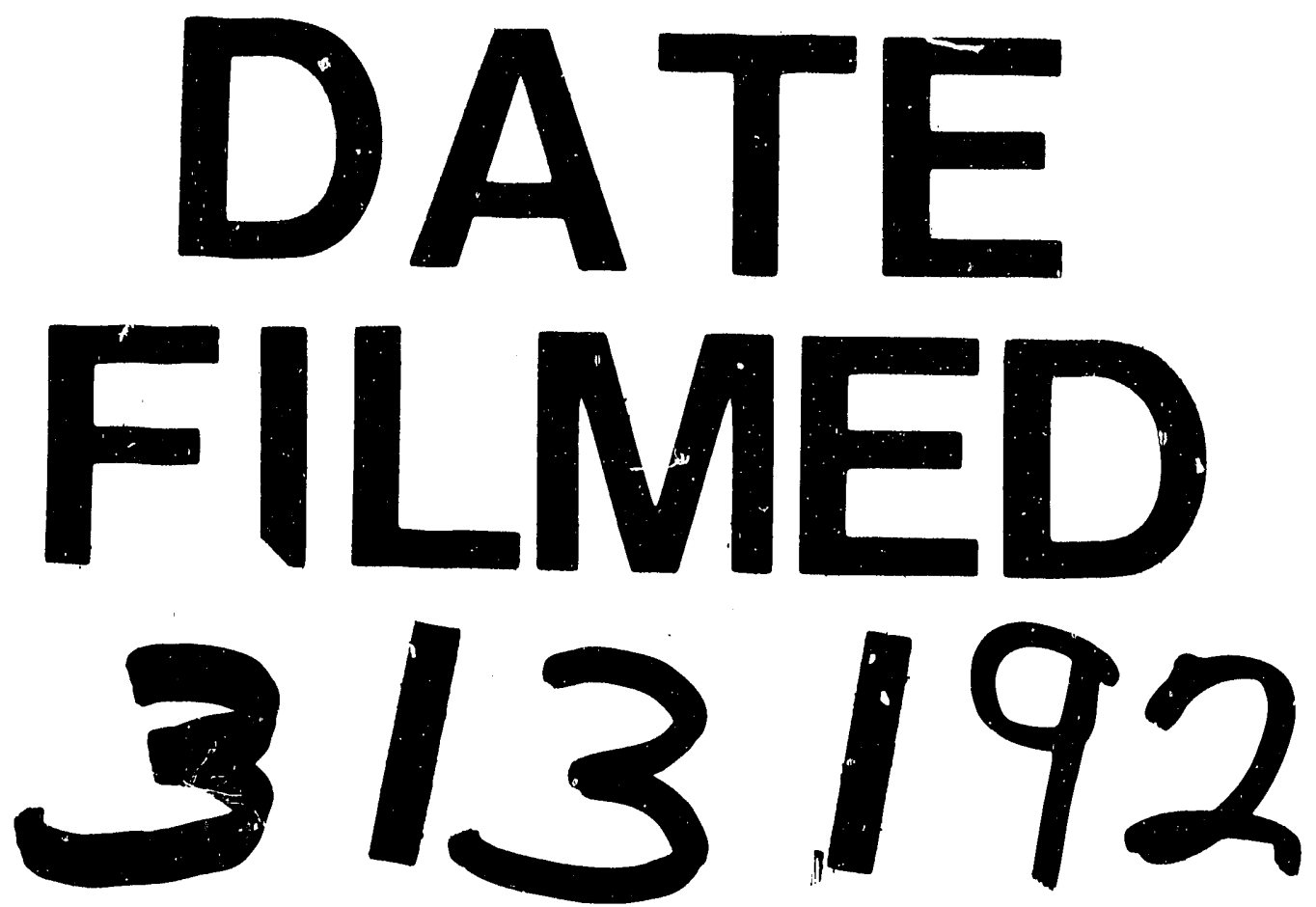

I

I. 